\title{
Influencia de la percepción de apoyo a la autonomía, la autoeficacia y la satisfacción académica en la intención de permanencia de estudiantado universitario
}

\author{
Influence of the Perceived Autonomy Support, Self-Efficacy, and Academic Satisfaction in \\ the Intentions of Permanence of University Students \\ Influência da percepção de apoio à autonomia, a autoeficácia e a satisfação acadêmica na \\ intenção de permanência de estudantes universitários
}

Paulina Barrientos-Illanes

Universidad de Concepción

Concepción, Chile

paulina.barrientos@uss.cl

(iD http://orcid.org/0000-0002-5413-1749

María Victoria Pérez-Villalobos

Universidad de Concepción

Departamento de Psicología

Concepción, Chile

marperez@udec.cl

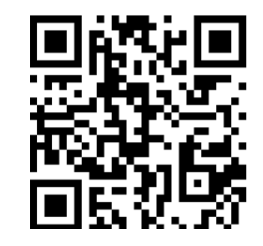

iD http://orcid.org/0000-0002-3697-7978

Jorge Vergara-Morales

Universidad de Las Américas

Facultad de Salud y Ciencias Sociales

Concepción, Chile

jvergaram@udla.cl

iD http://orcid.org/0000-0003-3655-813X

Alejandro Díaz-Mujica

Universidad de Concepción

Departamento de Psicología

Concepión, Chile

adiazm@udec.cl

http://orcid.org/0000-0002-3090-5463

Recibido • Received • Recebido: 13 / 06 / 2019

Corregido • Revised • Revisado: 14 / 01 / 2021

Aceptado • Accepted • Aprovado: 16 / 02 / 2021 
http://doi.org/10.15359/ree.25-2.5

http://www.una.ac.cr/educare

educare@una.ac.cr

Resumen: Diversos estudios ponen de manifiesto la necesidad de indagar los procesos psicológicos que intervienen en la permanencia universitaria. El objetivo del estudio fue estimar la influencia de la percepción de apoyo a la autonomía y la autoeficacia académica en la intención de permanencia de estudiantado universitario, evaluando el rol mediador de la satisfacción académica. Las personas participantes fueron 273 estudiantes de primer año, de una universidad privada de la región del Bío Bío, Chile. La influencia de los factores cognitivo-motivacionales se evaluó mediante análisis de senderos. El efecto mediador se evaluó utilizando el programa Mplus. Los resultados indicaron que la satisfacción académica media totalmente el efecto de la percepción de apoyo a la autonomía y de la percepción de autoeficacia académica sobre la intención de permanencia. Se concluye que los niveles positivos de satisfacción académica influyen positivamente en la intención de permanecer estudiando una carrera universitaria.

Palabras claves: Permanencia; satisfacción académica; análisis de senderos; análisis de mediación.

Abstract: Several studies show the need to investigate the psychological processes involved in university dropout. The study aimed to estimate the influence of perceived autonomy support and academic self-efficacy perception on the intention of permanence of university students, evaluating the mediating role of academic satisfaction. The participants were 273 first-year students from a private university in Bio Bio, Chile. The influence of cognitive-motivational factors was assessed through path analysis. The mediating effect was evaluated through the Mplus program. Results show that academic satisfaction fully mediates the effect of the perceived autonomy support and the perception of academic self-efficacy on the intention of permanence. It is concluded that the positive levels of academic satisfaction positively influence the intention to continue studying a university degree.

Keywords: Permanence; academic satisfaction; path analysis; mediation analysis.

Resumo: Vários estudos mostram a necessidade de investigar os processos psicológicos que intervêm na permanência de estudantes na universidade. O objetivo do estudo foi estimar a influência de fatores cognitivo-motivacionais na intenção de permanência de universitários, avaliando o papel mediador da satisfação acadêmica. Os participantes eram 273 calouros de uma universidade particular na região do Bío Bío, Chile. A influência dos fatores cognitivo-motivacionais foi avaliada pela análise de rota. O efeito mediador foi avaliado usando o programa Mplus. Os resultados indicaram que a satisfação acadêmica regula totalmente o efeito da percepção de apoio à autonomia e da percepção da autoeficácia acadêmica sobre a intenção de permanencia. Conclui-se que os níveis positivos de satisfação acadêmica influenciam positivamente a intenção de continuar estudando uma carreira universitária.

Palavras-chave: Permanência; satisfação acadêmica; análise de rota; análise de mediação. 


\section{Introducción}

En los últimos 30 años, la educación superior en Chile ha aumentado de forma exponencial el ingreso de estudiantes, configurándose un espacio social más heterogéneo que los anteriores, caracterizados por elites sociales con un vasto capital social y cultural, así como con una tradición familiar de estudios universitarios. En el contexto actual, un gran porcentaje de estudiantes corresponde a la primera generación familiar en asistir a la universidad, donde además los estratos socioeconómicos son variados y el capital sociocultural es más bajo que el de las elites sociales (Fonseca-Grandón, 2018). Si bien la democratización en el acceso a la educación superior ha significado un avance en términos de movilidad social, ha implicado un proceso de transformación social que no ha estado exento de dificultades. Para el caso de Chile, la deserción en primer año presenta niveles preocupantes, ya que alcanza en promedio un $30 \%$. La tasa de permanencia del estudiantado de pregrado durante el primer año universitario en el 2015, fue de un 71,3\%; en el año 2016 fue de 72,4\% y el año 2017 de 74\% (Servicio de Información de la Educación Superior [SIES], 2018).

La intención de abandono o de permanencia es entendida como la posibilidad que un estudiante evalúa respecto a descontinuar o continuar los estudios universitarios. La mayoría de los estudios se han basado en diferentes perspectivas y modelos de análisis, identificándose una tendencia actual de incorporar variables cognitivo-motivacionales para estudiar la permanencia académica (Arancibia Carvajal y Trigueros Cervantes, 2018; Velázquez Narváez y González Medina, 2017). Dentro de los factores cognitivo-motivacionales que se han asociado con el abandono y la permanencia en los estudios superiores, se han identificado la percepción de apoyo a la autonomía, la autoeficacia y la satisfacción académica. Los resultados han mostrado que cuando el estudiantado percibe altos niveles de autoeficacia académica, de apoyo a la autonomía y de satisfacción académica, menores son sus probabilidades de abandonar el sistema educativo y mayores son sus posibilidades de mejorar el desempeño académico (Antaramian, 2017; Jung y Lee, 2018; Ratsameemonthon et al., 2018; Wilkins-Yel et al., 2018; You, 2018).

La teoría de la autodeterminación plantea dos estilos motivacionales de enseñanza, desde los cuales las personas docentes influyen en la motivación del estudiantado: el estilo controlador y el estilo que apoya la autonomía. La adopción por parte de docentes de un estilo de apoyo a la autonomía frente a un estilo de control, no es suficiente para fomentar la motivación autónoma en sus estudiantes, ya que es necesario que estos grupos perciban el apoyo para actuar de forma voluntaria y sin presión externa (Reeve et al., 2014; Su y Reeve, 2010).

Se ha observado que el estudiantado que percibe mayor apoyo a la autonomía por parte del personal docente, y que por ende cuenta con un contexto que favorece la satisfacción de sus necesidades psicológicas básicas, presenta mayores niveles de autoeficacia académica, lo que 
http://doi.org/10.15359/ree.25-2.5

http://www.una.ac.cr/educare

educare@una.ac.cr

se evidencia en un aprendizaje caracterizado por un mayor interés, compromiso y satisfacción. Esto da lugar a comportamientos más autorregulados en el contexto académico, lo que se refleja en niveles positivos de bienestar y disfrute de la experiencia de aprendizaje (Oriol-Granado et al., 2017). Cuando el estudiantado tiene una alta percepción de apoyo a la autonomía por parte del personal docente, no solo presenta altos niveles de autoeficacia sino también altos niveles de satisfacción académica, lo que finalmente favorece la intención de permanecer estudiando una carrera (titulación) universitaria (Bernardo Gutiérrez et al., 2018; Haerens et al., 2018).

La satisfacción académica se ha conceptualizado como "un componente cognitivo del bienestar psicológico que refiere a las valoraciones que las personas realizan al comparar sus aspiraciones con sus logros alcanzados" (Medrano et al., 2014, citado por Vergara-Morales et al., 2018, p. 100). Constituye también un referente para la satisfacción general en la vida del estudiantado (Meštrović, 2017). La satisfacción académica se traduce en el estado de ánimo que tiene lugar a partir de la interpretación que estudiantes realizan de sus capacidades en el contexto académico (Cabana et al., 2016). El estudiantado con altos niveles de satisfacción académica presenta una mejor integración de las demandas académicas y del contexto universitario, así como mayores niveles de satisfacción con la vida, lo que se refleja en la permanencia y continuidad de los estudios universitarios (Antaramian, 2017; Duffy et al., 2011; Wilkins-Yel et al., 2018).

Por su parte, autoeficacia académica tiene una influencia inversa en la intención de abandono del estudiantado y en la satisfacción académica (Casanova et al., 2018). Se ha identificado una relación directa no significativa con la intención de permanencia (Navarro Charris et al., 2017). Desde una perspectiva conceptual, la autoeficacia académica corresponde a las creencias que el estudiantado tiene sobre sus propias capacidades para desempeñarse en el ámbito académico. Es decir, se refiere a los juicios sobre la capacidad para organizar y ejecutar las acciones necesarias para obtener éxito académico (Abello Riquelme et al., 2012; Ornelas et al., 2012). Se considera como un constructo que puede variar en función de las tareas y de las circunstancias ambientales. Esta característica de permeabilidad implica que es susceptible de ser mejorada con el apoyo docente, tal como lo sugieren diversos estudios (Hosseini et al., 2018; Larose et al., 2018; Metcalf y Wiener, 2018; Pennington et al., 2018).

Sobre la base de los antecedentes revisados, se consideró relevante estudiar cómo la percepción de apoyo a la autonomía, la percepción de autoeficacia y la satisfaccion académica conducen a mayores niveles de intención de permanencia en estudiantes de educación superior, identificando el rol medidador de la satisfacción académica. Por lo tanto, el objetivo del estudio fue estimar la influencia de la percepción de apoyo a la autonomía y la percepción de autoeficacia académica en la intención de permanencia del estudiantado universitario, evaluando el rol mediador de la satisfacción académica. 


\section{Método}

\section{Diseño}

La investigación empleó un diseño cuantitativo, de corte transversal, no experimental, utilizó una estrategia asociativa de carácter explicativa, ya que se sometieron a prueba "modelos acerca de las relaciones existentes entre un conjunto de variables, tal y como se derivan de una teoría subyacente" (Ato et al., 2013, p. 1052).

\section{Participantes}

Las personas participantes fueron un total de 273 estudiantes de primer año de una universidad privada de la región del Bío Bío, Chile, procedentes de cuatro carreras: Psicología (22\%), Fonoaudiología (13\%), Tecnología Médica (38\%) y Terapia Ocupacional (27\%). Un 34,7\% de estudiantes fueron hombres y un 65,3\% fueron mujeres. El rango de edad varió entre los 18 y 30 años, con una edad media de 19,8 años. La selección de la muestra se realizó utilizando un muestreo no probabilístico por conveniencia.

\section{Instrumentos}

Escala de percepción de apoyo a la autonomía (versión abreviada): evalúa la percepción del estudiantado sobre el grado en que el personal docente promueve su autonomía. Se compone de 6 ítems (p.ej.: Mis profesores me dan opciones y posibilidades de hacer elecciones) los que se responden en una escala Likert de 7 puntos ( $1=$ en total desacuerdo a $7=$ muy de acuerdo). En este estudio se utilizó una versión adaptada al español con adecuados niveles de fiabilidad y validez (Matos et al., 2018).

Escala de percepción de autoeficacia académica: este instrumento mide"las expectativas de autoeficacia en situaciones específicas del contexto educativo" (del Valle et al., 2018, p. 99). Se compone de 10 ítems (p.ej.: "Tengo la convicción de que puedo obtener excelentes notas en las pruebas") (del Valle et al., 2018, columna 2, fila, 7, p. 102), que se respondieron en una escala tipo Likert de 7 puntos ( 1 = totalmente en desacuerdo; 7 = totalmente de acuerdo). La suma total de las puntuaciones indicó el grado de autoeficacia académica percibida por el estudiantado. En este estudio se utilizó una versión adaptada al español con adecuados niveles de fiabilidad y validez (Dominguez Lara, 2014).

Escala de satisfacción académica: evalúa el grado en el que el estudiantado se siente satisfecho en general en sus estudios. Está compuesta por 7 ítems (p.ej.: "Estoy satisfecho(a) con estar cursando esta carrera"), con una escala de respuesta tipo Likert de siete alternativas ( 1 = totalmente en desacuerdo a $7=$ totalmente desacuerdo). El grado de satisfacción se obtuvo a partir de la media total. La escala original reporta una estructura unifactorial con adecuados niveles de consistencia interna. En este estudio se utilizó una versión adaptada al español con adecuados niveles de fiabilidad y validez (Medrano et al., 2014). 
http://doi.org/10.15359/ree.25-2.5

http://www.una.ac.cr/educare

educare@una.ac.cr

Escala de intención de permanencia: se constituyó de un ítem que midió la intención del alumnado de continuar estudiando la misma carrera. El ítem se respondió en una escala Likert de 7 puntos ( 1 = "totalmente en desacuerdo"; 7 = "totalmente de acuerdo").

\section{Procedimiento}

La investigación se realizó siguiendo las consideraciones éticas de la Asociación Americana de Psicología (APA). El proceso de recolección de los datos se realizó en las aulas del estudiantado, y se consideró, previamente, la aprobación de las autoridades. El estudiantado participó voluntariamente de la investigación. Además, se estableció el uso confidencial de los datos.

\section{Análisis de los datos}

El análisis descriptivo se realizó calculando la media y la desviación estándar. Para evaluar la consistencia interna de las puntuaciones se utilizó el alfa de Cronbach, considerando un límite inferior de $a=0.70$ para indicar una fiabilidad aceptable. Finalmente, se utilizó el coeficiente de correlación RHO de Spearman para la evaluar la asociación entre las variables.

Se utilizó el análisis de senderos para evaluar el ajuste del modelo hipotético a los datos observados. Para la elaboración del modelo hipotético se consideró como variable exógena la percepción de apoyo a la autonomía. Como variables endógenas mediadoras se incluyeron la percepción de autoeficacia académica y la satisfacción académica. La variable endógena de resultado fue la intención de permanencia.

El modelo de senderos consideró las siguientes hipótesis: (1) La percepción de apoyo a la autonomía tiene un efecto positivo y directo sobre la satisfacción académica y la percepción de autoeficacia; (2) la percepción de autoeficacia tiene un efecto positivo y directo sobre la satisfacción académica; y (3) la satisfacción académica tiene un efecto positivo y directo sobre la intención de permanencia.

Para evaluar el ajuste del modelo de relaciones hipotetizado, se utilizó el método de estimación WLSMV, ya que la variable endógena de resultado se consideró de nivel ordinal. La bondad de ajuste se estimó considerando los índices y criterios:

a) $X^{2}$ : valores no significativos indican un buen ajuste; b) $X^{2} / d f$ : un buen ajuste se indica con valores inferiores a 2; c) índice de ajuste comparativo (CFI) y el índice de Tucker-Lewis (TLI): un ajuste aceptable se indica a través de valores $\geq .90$ y un buen ajuste está determinado por valores $\geq .95$; d) error cuadrático medio de aproximación (RMSEA): un ajuste aceptable está determinado por valores $\leq .08$ (IC del 90\% $\leq .10)$ y un buen ajuste se indica mediante valores $\leq .05$ (IC del 90\% < .08) (Burnham y Anderson, 1998; Hu y Bentler, 1999 en VergaraMorales et al., 2018, p. 102). 
http://doi.org/10.15359/ree.25-2.5

El análisis de mediación implicó la evaluación de los siguientes efectos indirectos: (1) el efecto de la percepción de apoyo a la autonomía en la intención de permanencia, a través la satisfacción académica; y (2) el efecto de la percepción de autoeficacia académica en la intención de permanencia, a través de la satisfacción académica. Los análisis fueron realizados utilizando el programa Mplus.

\section{Resultados}

\section{Análisis descriptivo y correlacional}

La Tabla 1 presenta el análisis descriptivo y correlacional de las variables sometidas a estudio. Al respecto, se observa que la intención de permanencia $(M=6.56)$ y la satisfacción académica $(M=5.97)$ tuvieron las puntuaciones medias más altas. Por otro lado, los valores de desviación típica fueron cercanos a 1.00, lo que indica niveles adecuados de dispersión de los datos. Todas las variables presentaron puntuaciones con una adecuada consistencia interna, ya que los valores de los coeficientes a fueron igual o mayores a 0.90. Finalmente, las correlaciones entre las variables fueron positivas y significativas, destacándose la asociación entre la satisfacción académica y la intención de permanencia del estudiantado.

Tabla 1: Análisis descriptivo y correlacional

\begin{tabular}{lccccccc}
\hline \multicolumn{1}{c}{ Variables } & M & D.T. & a & 1 & 2 & 3 & 4 \\
\hline $\begin{array}{l}\text { 1. Percepción de apoyo a la autonomía } \\
\text { 2.14 }\end{array}$ & 1.12 & 0.90 & 1.00 & $0.40^{* *}$ & $0.51^{* *}$ & $0.19^{* *}$ \\
2. Percepción de autoeficacia académica & 5.37 & 0.96 & 0.92 & & 1.00 & $0.54^{* *}$ & $0.24^{* *}$ \\
3. Satisfacción académica & 5.97 & 0.89 & 0.90 & & & 1.00 & $0.54^{* *}$ \\
4. Intención de permanencia & 6.56 & 0.98 & - & & & & 1.00 \\
\hline
\end{tabular}

** $\mathrm{p}<0.01$

Nota: Elaboración propia.

\section{Análisis de senderos}

En la Tabla 2 se muestra que los valores de los índices indican un buen ajuste del modelo hipotético a los datos observados. Por lo tanto, los datos apoyan el efecto positivo y directo de la percepción de apoyo a la autonomía sobre la satisfacción académica $(\beta=0.33)$ y la percepción de autoeficacia académica $(\beta=0.36)$. Además, se observó que la percepción de autoeficacia 
http://doi.org/10.15359/ree.25-2.5

http://www.una.ac.cr/educare

educare@una.ac.cr

académica tuvo un efecto positivo y directo sobre la satisfacción académica ( $\beta=0.39)$. Finalmente, solo la satisfacción académica se relacionó positivamente con la intención de permanencia $(\beta=0.59)$ (ver Figura 1).

Tabla 2: Índices de ajuste del modelo hipotético

\begin{tabular}{ccccccccc}
\hline & $\mathrm{x}^{2}$ & $\mathrm{gl}$ & $\mathrm{p}$ & $\mathrm{x}^{2} / \mathrm{df}$ & $\mathrm{TLI}$ & $\mathrm{CFI}$ & RMSEA & IC 90\% \\
\hline Modelo hipotético & 1.88 & 2 & 0.39 & 0.94 & 1.00 & 1.00 & 0.00 & $0.00-0.12$ \\
\hline
\end{tabular}

Nota: Elaboración propia.

Figura 1: Modelo de senderos

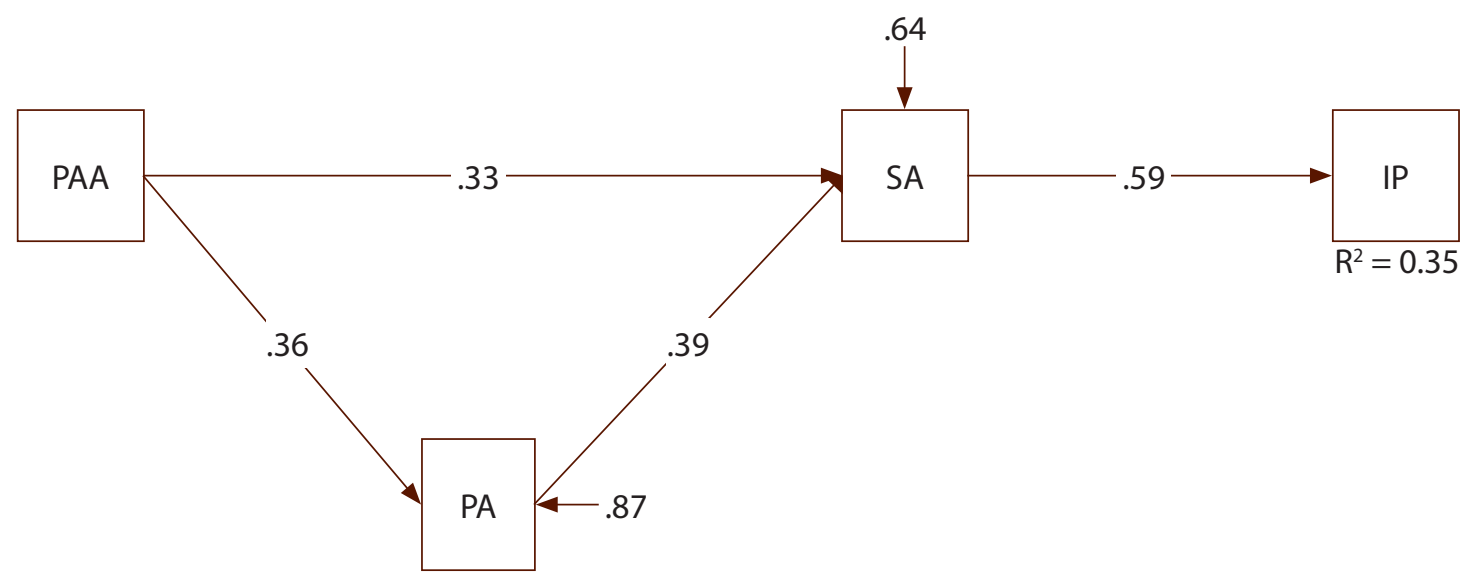

PAA = Percepción de apoyo a la autonomía; PA = Percepción de autoeficacia; SA = Satisfacción académica; IP = Intención de permanencia.

Nota: Elaboración propia

\section{Análisis de mediación}

En la Tabla 3 se muestra que la percepción de apoyo a la autonomía tuvo un efecto positivo e indirecto $(\beta=0.20)$ sobre la intención de permanencia, mediado totalmente por la satisfacción académica. Por otro lado, se observó que la percepción de autoeficacia académica también tuvo un efecto positivo e indirecto $(\beta=0.23)$ sobre la intención de permanencia, totalmente mediado por la satisfacción académica. 
Tabla 3: Análisis de mediación

\begin{tabular}{ccll}
\hline Variables exógenas & Variable Mediadora & Efectos & IP \\
\hline PAA & & Directo & 0.00 \\
& SA & Indirecto & $0.20^{* *}$ \\
PA & & Directo & 0.00 \\
& SA & Indirecto & $0.23^{* *}$ \\
\hline
\end{tabular}

PAA = Percepción de apoyo a la autonomía; PA = Percepción de autoeficacia académica;

$\mathrm{SA}=$ Satisfacción académica; IP = Intención de permanencia; ${ }^{* *} \mathrm{p}<0.01$

Nota: Elaboración propia

\section{Discusión y conclusiones}

El objetivo del estudio fue estimar la influencia de la percepción de apoyo a la autonomía y la percepción de autoeficacia académica en la intención de permanencia de estudiantes universitarios, evaluando el rol mediador de la satisfacción académica.

Los resultados reflejan adecuadamente las relaciones entre las variables del modelo de senderos inicialmente estimado. Considerando la teoría de la autodeterminación, el modelo de senderos evidenció que la percepción de un estilo motivacional docente promotor de espacios de autonomía en el proceso de aprendizaje favorece tanto la satisfacción con el estudiar una carrera universitaria, con las asignaturas y las clases, como los juicios positivos respecto de las propias capacidades para desempeñarse en el ámbito académico. Estos resultados confirman el planteamiento previo (Bernardo Gutiérrez et al., 2018; Haerens et al., 2018; Oriol-Granado et al., 2017) de que la percepción de un estilo motivacional docente que apoya la autonomía genera condiciones de aprendizaje favorecedoras para la satisfacción con las experiencias educativas y percepciones positivas respecto a las propias capacidades académicas.

El modelo de senderos evidenció también que la valoración positiva de las propias capacidades académicas favorece niveles positivos de satisfacción con el estudiar una carrera universitaria, con las asignaturas y las clases. Estos resultados coinciden con estudios anteriores (Casanova et al., 2018; Medrano et al., 2014), donde se encontró que la percepción que el estudiantado universitario tiene de sus capacidades académicas, determina, en gran medida, el nivel de satisfacción con que vivencia el estudiar una carrera universitaria, las clases y las relaciones sociales que allí se establecen. 
http://doi.org/10.15359/ree.25-2.5

http://www.una.ac.cr/educare

educare@una.ac.cr

Se observó que altos niveles de satisfacción académica asociados a estudiar una carrera universitaria generan un contexto que favorece la intención de permanecer estudiando la carrera. Estos resultados coinciden con los hallazgos de investigaciones anteriores (Antaramian, 2017; Duffy et al., 2011; Wilkins-Yel et al., 2018), las que señalan que altos niveles de satisfacción académica se asocian a una mayor intención de permanencia y continuidad en los estudios universitarios.

A través del análisis de mediación, se determinó un rol totalmente mediador de la satisfacción académica, en la relación entre percepción de apoyo a la autonomía e intención de permanencia. Es decir, cuando la satisfacción académica intervino, el efecto de la percepción de un comportamiento docente que apoya la autonomía genera mayores niveles en la intención de permanecer estudiando una carrera universitaria. Estos resultados se ajustan a los hallazgos de estudios anteriores (Bernardo Gutiérrez et al., 2018; Haerens et al., 2018), pues para el caso de la muestra de estudio, se observó que cuando el estudiantado percibe espacios de autonomía por parte del personal docente, presenta también altos niveles de bienestar asociados a las experiencias académicas, lo que finalmente favorece la intención de permanecer estudiando una carrera universitaria.

Por otro lado, la satisfacción académica tuvo también un rol totalmente mediador en la relación entre percepción de autoeficacia académica e intención de permanencia. Es decir, a través de altos niveles de satisfacción con la experiencia de aprendizaje, la valoración positiva de las propias capacidades académicas afectó indirectamente la intención de permanecer estudiando la carrera universitaria. Este trabajo coincide con estudios anteriores (Navarro Charris et al., 2017) donde no se encontró evidencia suficiente para aceptar una relación directa entre la percepción de autoeficacia académica y la intención de permanencia. De esta manera, la satisfacción académica constituye un mecanismo psicológico que media la relación de la percepción de autoeficacia académica sobre la intención de permanencia.

Estos resultados tienen implicancias prácticas, tanto para las instituciones de educación superior como para el personal docente, ya que los resultados muestran que cuando el estudiantado percibe un comportamiento docente que apoya el desarrollo de su autonomía en el aprendizaje, experimenta altos niveles de satisfacción con las experiencias académicas, lo cual facilita su intención de permanecer estudiando una carrera. También, la percepción del estilo motivacional docente que a poya la autonomía favorece la satisfacción con las experiencias académicas y la percepción positiva de las propias capacidades académicas. El que estudiantes valoren, de forma positiva, sus propias capacidades académicas, favorece el logro de altos niveles de satisfacción académica asociados a la experiencia universitaria, lo que, finalmente, se asocia a la intención de permanecer estudiando una carrera. 
http://doi.org/10.15359/ree.25-2.5

Se debe advertir que la muestra únicamente incluyó estudiantes de primer año de una universidad privada de la región del Bío Bío, Chile, seleccionados a través de un muestreo no probabilístico. Para hacer más generalizables los resultados, es recomendable que futuras investigaciones consideren estudiantes de distintos niveles académicos, carreras yuniversidades.

\section{Declaración de derivación y procedencia}

Este artículo es parte del Proyecto CONICYT-FONDECYT N 1161502 titulado"Modelo explicativo de la permanencia y el abandono de los estudios universitarios, basado en procesos cognitivosmotivacionales".

\section{Declaración de Material complementario}

Este artículo tiene disponible, como material complementario:

-La versión preprint del artículo en https://doi.org/10.5281/zenodo.3661346

\section{Referencias}

Abello Riquelme, R., Díaz Mujica, A., Pérez Villalobos, M. V., Almeida, L. S., Lagos Herrera, I., González Puentes, J. y Strickland, B. (2012). Vivencias e implicación académica en estudiantes universitarios: Adaptación y validación de escalas para su evaluación. Estudios Pedagógicos, 38(2), 7-19. https://doi.org/10.4067/S0718-07052012000200001

Antaramian, S. (2017). The importance of very high life satisfaction for students' academic success. Cogent Education, 4(1), 1-10. https://doi.org/10.1080/2331186X.2017.1307622

Ato, M., López, J. J. y Benavente, A. (2013). Un sistema de clasificación de los diseños de investigación en psicología. Anales de Psicología, 29(3), 1038-1059. https://doi.org/10.6018/ analesps.29.3.178511

Bernardo Gutiérrez, A. B., Fernández-Castañon, A. C., García, M. E., Fernández González, A., Solano Pizarro, P. y Agulló Tomás, E. (2018). Variables relacionadas con la intención de abandono universitario en el periodo de transición. Revista d'Innovació Docent Universitària, 10, 122130. https://doi.org/10.1344/RIDU2018.10.11

Cabana, S. R., Cortés, F. H., Vega, D. L. y Cortés, R. A. (2016). Análisis de la fidelización del estudiante de ingeniería con su centro de educación superior: Desafíos de gestión educacional. 
http://doi.org/10.15359/ree.25-2.5

http://www.una.ac.cr/educare

educare@una.ac.cr

Formación Universitaria, 9(6), 93-104. https://doi.org/10.4067/\$0718-50062016000600009

Arancibia Carvajal, R. y Trigueros Cervantes, C. (2018). A proximaciones a la deserción universitaria en Chile. Educação e Pesquisa, 44. https://doi.org/10.1590/s1678-4634201708165743

Casanova, J. R., Fernández-Castañon, A. C., Núñez Pérez, J. C., Bernardo Gutiérrez, A. B. y Almeida, L. S. (2018). Abandono no Ensino Superior: Impacto da autoeficácia na intenção de abandono. Revista Brasileira de Orientação Profissional, 19(1), 41-49. https://doi. org/10.26707/1984-7270/2019v19n1p41

del Valle, M., Díaz, A., Pérez, M. V., Vergara, J. (2018). Análisis factorial confirmatorio escala autoeficacia percibida en situaciones académicas (EAPESA) en universitarios chilenos. Revista Iberoamericana de Diagnóstico y Evaluación - e Avaliação Psicológica. RIDEP, 4(49), 97-106. https://doi.org/10.21865/RIDEP49.4.08

Dominguez Lara, S. (2014). Autoeficacia para situaciones académicas en estudiantes universitarios peruanos: Un enfoque de ecuaciones estructurales. Revista de Psicología, 4(4), 45-53. http://revistas.ucsp.edu.pe/index.php/psicologia/issue/view/4

Duffy, R. D., Allan, B. A. y Dik, B. J. (2011). The presence of a calling and academic satisfaction: Examining potential mediators. Journal of Vocational Behavior, 79(1), 74-80. https://doi. org/10.1016/j.jvb.2010.11.001

Fonseca-Grandón, G. R. (2018). Trayectorias de permanencia y abandono de estudios universitarios: Una aproximación desde el currículum y otras variables predictoras. Educación y Educadores, 21(2), 239-256. https://doi.org/10.5294/edu.2018.21.2.4

Haerens, L., Vansteenkiste, M., De Meester, A., Delrue, J., Tallir, I., Vande Broek, G., Goris, W. y Aelterman, N. (2018). Different combinations of perceived autonomy support and control: Identifying the most optimal motivating style. Physical Education and Sport Pedagogy, 23(1), 16-36. https://doi.org/10.1080/17408989.2017.1346070

Hosseini, F., Hossein Maktabi, G., Shehni Yailagh, M. y Haji Yakhchali, A. (2018). The effects of teaching reflective thinking model on academic self-efficacy, achievement motivation, intention certainty and reflective thinking of the of Farhangyan University students in Ahvaz. Ahvaz, Iran. Amazonia Investiga, 7(13), 401-418. https://amazoniainvestiga.info/ index.php/amazonia/article/view/581/550

Jung, Y. y Lee, J. (2018). Learning engagement and persistence in massive open online courses (MOOCS). Computers \& Education, 122, 9-22. https://doi.org/10.1016/j. compedu.2018.02.013

Larose, S., Boisclair-Châtteauvert, G., de Wit, D. J., Dubois, D., Erdem, G. y Lipman, E. L. (2018). How mentor support interacts with mother and teacher support in predicting youth academic adjustment: An investigation among youth exposed to big brothers big sisters of Canada

12 Paulina Barrientos-Illanes, María Victoria Pérez-Villalobos, Jorge Vergara-Morales y Alejandro Díaz-Mujica

Los artículos de la Revista Electrónica Educare del Centro de Investigación y Docencia en Educación de la Universidad Nacional, Costa Rica, se comparten bajo términos de la Licencia Creative Commons: Reconocimiento, No Comercial, Sin Obra Derivada 3.0 Costa Rica. Las autorizaciones adicionales a las aquí delimitadas se pueden obtener en el correo: educare@una.cr 
http://doi.org/10.15359/ree.25-2.5

programs. The Journal of Primary Prevention, 39(3), 205-228. https://doi.org/10.1007/ $\underline{\text { s10935-018-0509-8 }}$

Matos, L., Reeve, J., Herrera, D. y Claux, M. (2018). Students' agentic engagement predicts longitudinal increases in ššš) for first-year university student. Electronic Journal of Research in Educational Psychology, 12(2), 541-562. http://www.investigacion-psicopedagogica. org/revista/new/english/ContadorArticulo.php?906

Medrano, L. A., Fernández Liporace, M. y Pérez, E. (2014). Computerized Assesment System for Academic Satisfaction (ASAS) for first-year university student. Electronic Journal of Research in Educational Psychology, 12(2), 541-562. http://www.investigacion-psicopedagogica. org/revista/new/english/ContadorArticulo.php?906

Meštrović, D. (2017). Service quality, students' satisfaction and behavioural intentions in STEM and IC higher education institutions. Interdisciplinary Description of Complex Systems, 15(1), 66-77. https://doi.org/10.7906/indecs.15.1.5

Metcalf, D. A. y Wiener, K. K. K. (2018). Academic self-efficacy in a twenty-first-century australian university: strategies for first-generation students. Higher Education Research and Development, 37(7), 1472-1488. https://doi.org/10.1080/07294360.2018.1484705

Navarro Charris, N. E., Redondo Bilbao, O. E., Contreras Salinas, J. A., Romero Díaz, C. H. y D'Andreis Zapata, A. C. (2017). Permanencia y deserción versus autoeficacia de estudiantes universitarios: Un desafío de la calidad educativa. Revista Lasallista de Investigación, 14(1), 198-206. https://doi.org/10.22507/rli.v14n1a17

Oriol-Granado, X., Mendoza-Lira, M., Covarrubias-Apablaza, C.-G. y Molina-López, V.M. (2017). Emociones positivas, apoyo a la autonomía y rendimiento de estudiantes universitarios: El papel mediador del compromiso académico y la autoeficacia. Revista de Psicodidáctica, 22(1), 45-53. https://doi.org/10.1387/RevPsicodidact.14280

Ornelas, M., Blanco, H., Gastélum, G. y Chávez, A. (2012). Autoeficacia percibida en la conducta académica de estudiantes universitarias. Formacion Universitaria, 5(2), 17-26. https://doi. org/10.4067/S0718-50062012000200003

Pennington, C. R., Bates, E. A., Kaye, L. K. y Bolam, L. T. (2018). Transitioning in higher education: An exploration of psychological and contextual factors affecting student satisfaction. Journal of Further and Higher Education, 42(5), 596-607. https://doi.org/10.1080/030987 7X.2017.1302563

Ratsameemonthon, L., Ho, R., Tuicomepee, A. y Blauw, J. N. (2018). Influence of achievement goals and academic self-efficacy on academic achievement of thai undergraduate students : 
http://doi.org/10.15359/ree.25-2.5

http://www.una.ac.cr/educare

educare@una.ac.cr

Across non procrastinators and procrastinators. Electronic Journal of Research in Education Psychology, 16(45), 243-271. https://doi.org/10.25115/ejrep.v16i45.2093

Reeve, J. M, Vansteenkiste, M., Assor, A., Ahmad, I., Cheon, S. H., Jang, H., Kaplan, H., Moss, J. D., Stokke, B. y Wang, J. (2014). The beliefs that underlie autonomy-supportive and controlling teaching: A multinational investigation. Motivation and Emotion, 38(1), 93-110. https:// doi.org/10.1007/s11031-013-9367-0

Servicio de Información de Educación Superior. (2018). Informe de retención de $1^{\text {er }}$ año de pregrado. Cohorte 2013-2017. Autor.

Su, Y.-L. y Reeve, J. (2010). A meta-analysis of the effectiveness of intervention programs designed to support autonomy. Educational Psychology Review, 23(1), 159-188. https:// doi.org/10.1007/s10648-010-9142-7

Velázquez Narváez, Y. y González Medina, M. (2017). Factores asociados a la permanencia de estudiantes universitarios: Caso UAMM-UAT. Revista de la Educación Superior, 46(184), 117 138. https://doi.org/10.1016/j.resu.2017.11.003

Vergara-Morales, J., del Valle, M., Díaz, A. y Pérez, M. V. (2018). Adaptación de la escala de satisfacción académica en estudiantes universitarios chilenos. Psicología Educativa, 24(2), 99-106. https://doi.org/10.5093/psed2018a15

Wilkins-Yel, K. G., Roach, C. M.L., Tracey, T. J. G. y Yel, N. (2018). The effects of career adaptability on intended academic persistence: The mediating role of academic satisfaction. Journal of Vocational Behavior, 108, 67-77. https://doi.org/10.1016/j.jvb.2018.06.006

You, J. W. (2018). Testing the three-way interaction effect of academic stress, academic selfefficacy, and task value on persistence in learning among Korean college students. Higher Education, 76(5), 921-935. https://doi.org/10.1007/s10734-018-0255-0 